SCIPP-00/08, KAIST-TH 00/06, KIAS-P00015, hep-ph/0004101

\title{
Fermion Electric Dipole Moments in Supersymmetric Models with R-parity Violation
}

\author{
Kiwoon Choi ${ }^{a, b}$, Eung Jin Chun ${ }^{c}$ and Kyuwan Hwang ${ }^{b}$ \\ ${ }^{a}$ Santa Cruz Institute for Particle Physics, Santa Cruz, CA 95064, U. S. A. \\ ${ }^{b}$ Korea Advanced Institute of Science and Technology, Taejeon 305-701, Korea \\ ${ }^{c}$ Korea Institute for Advanced Study, Seoul 130-012, Korea
}

\begin{abstract}
We analyze the electron and neutron electric dipole moments induced by R-parity violating interactions in supersymmetric models. It is pointed out that dominant contributions can come from one-loop diagrams involving both the bilinear and trilinear R-parity odd couplings, leading to somewhat severe constraints on the products of those couplings.
\end{abstract}

\section{INTRODUCTION}

Fermion dipole moments provide severe constraints on possible new physics beyond the Standard Model. The foremost example is the electric dipole moment (EDM) of the electron or neutron whose current experimental bound is given, respectively, by [1.2]

$$
\begin{aligned}
& \left|d_{e}\right| \leq 4.3 \times 10^{-27} e \mathrm{~cm} \\
& \left|d_{n}\right| \leq 6.3 \times 10^{-26} e \mathrm{~cm}
\end{aligned}
$$

In the Minimal Supersymmetric Standard Model (MSSM), apart from the CKM phase, CP violation can arise from additional complex parameters describing the soft supersymmetry (SUSY) breaking terms in the effective Lagrangian. Such complex soft parameters generate a light fermion EDM through one-loop diagrams involving sparticles (squarks/sleptons and/or gauginos). The current bounds on the neutron and electron EDM constrain the phases of soft parameters to be less than $\mathcal{O}\left(10^{-2}\right)$, or the sfermion masses to be larger than $\mathcal{O}(1) \mathrm{TeV}$ [3] unless there are cancellations among various contributions [ [0].

When R-parity conservation is not assumed, the MSSM allows additional lepton number (L) or baryon number (B) violating interactions. In this case, one usually invokes other type of (either unbroken or spontaneously broken) symmetry which would ensure that R-parity violating couplings are small enough to avoid too fast proton decay and too large neutrino masses [5]. Imposing a symmetry which would forbid (or highly suppress) B-violating couplings while allowing non-zero L-violating couplings is an attractive possibility since then an interesting pattern of neutrino masses and mixing can be generated [6]. Furthermore these 
couplings can provide a new source of $\mathrm{CP}$ violation, and thus the EDMs of light fermions [7.8].

The quark and lepton EDMs arising from the R-parity violating Yukawa couplings have been studied recently in Refs. [0,8]. To construct effective dipole moment operator, it is required to have not only the chirality-flip but also the insertion of even number of R-parity violating couplings. In other words, the resulting fermion EDMs involve some powers of $\lambda \lambda$ and/or $\lambda \lambda^{*}$ as well as some fermion masses or chirality-flipping Yukawa couplings [9] where $\lambda$ denotes a generic R-parity violating Yukawa coupling. At one-loop level, EDM diagrams involving $\lambda \lambda$ exist. However such diagrams are suppressed by additional Majorana neutrino (or sneutrino) mass which would compensates the $L=2$ of $\lambda \lambda$ in the EDM coefficients, and thus are negligibly small. It has been verified that important contributions can arise from two-loop diagrams involving the insertion of quark or lepton mass inside the loop and also the products of R-parity violating couplings such as $\lambda \lambda^{*}[7]$ or $\lambda^{2} \lambda^{* 2}$ [8].

In this paper, we wish to point out that sizable EDMs can arise at one-loop level in the presence of both the bilinear and trilinear R-parity violating interactions. Let us recall that generic R-parity violating superpotential includes the bilinear Higgsino-lepton mixing term

$$
W \ni \mu_{i} L_{i} H_{2} \text {, }
$$

where $L_{i}(i=1,2,3)$ are the lepton $S U(2)$ doublets, and $H_{2}$ is the Higgs doublet with hypercharge $Y=1 / 2$. There exist also R-parity violating scalar bilinears in the soft SUSY breaking potential:

$$
V \ni B_{i} L_{i} H_{2}+m_{L_{i} H_{1}}^{2} L_{i} H_{1}^{\dagger}
$$

where $H_{1}$ is the Higgs doublet with $Y=-1 / 2$. These bilinear scalar terms induce nonzero vacuum expectation values $(\mathrm{VEVs})$ of sneutrino fields:

$$
\left\langle\tilde{\nu}_{i}\right\rangle \propto B_{i}^{*}\left\langle H_{2}^{0}\right\rangle+m_{L_{i} H_{1}}^{2 *}\left\langle H_{1}^{0}\right\rangle .
$$

The presence of these bilinear couplings yields a mass mixings between the R-parity even and odd particles, which are described by the sneutrino VEVs $\left\langle\tilde{\nu}_{i}\right\rangle$ and the Higgsino-lepton mixing parameter $\mu_{i}$. Since they are R-parity odd and also flips the fermion chirality without involving additional particles in the interaction vertex, these mass mixings are more efficient than the R-parity violating Yukawa coupling $\lambda$ for generating fermion EDMs. We will see that there exist one-loop diagrams for fermion EDMs which are proportional to the products of bilinear and trilinear R-parity odd couplings, e.g. $\left\langle\tilde{\nu}_{i}\right\rangle \lambda$ or $\mu_{i}^{*} \lambda$. Such diagrams are not suppressed by additional powers of small fermion masses or Yukawa couplings, and thus can provide strong limits on these product of R-parity odd couplings.

The organization of this paper is as follows. In Section II, we define the basis on which our analysis is performed and summarize how to diagonalize the mixings between the R-parity even and odd particles which are induced by the bilinear R-parity violation. In Section III, we classify and compute the main contributions to the quark and lepton EDMs involving both the bilinear and trilinear R-parity violating couplings. The calculational details are presented in the Appendix, and the bounds on couplings from the electron and neutron EDMs are summarized in Tables 1 and 2 under the assumption that those couplings take generic complex values. We conclude in Section IV. 


\section{PARTICLE MIXINGS INDUCED BY $R$ PARITY VIOLATION}

For comparison with experiments, it is convenient to work in the quark and lepton mass eigenbasis. In this prescription, we leave the neutrinos in the charged lepton mass eigenbasis since the mechanism of neutrino mass generation is quite different from that of other fermions. R-parity violation under consideration can be a major source of nonzero neutrino masses, and then the charged lepton mass eigenbasis is particularly useful for analyzing neutrino mass matrix. In the quark and charged lepton mass eigenbasis, the full superpotential of the MSSM fields including generic R-parity violating couplings is given by

$$
\begin{aligned}
W & =\mu H_{1} H_{2}+h_{i}^{e} H_{1} L_{i} E_{i}^{c}+h_{i}^{d}\left(H_{1}^{0} D_{i} D_{i}^{c}-H_{1}^{-} V_{i j}^{\dagger} U_{j} D_{i}^{c}\right)+h_{i}^{u}\left(H_{2}^{0} U_{i} U_{i}^{c}-H_{2}^{+} V_{i j} D_{j} U_{i}^{c}\right) \\
& +\epsilon_{i} \mu L_{i} H_{2}+\frac{1}{2} \lambda_{i j k} L_{i} L_{j} E_{k}^{c}+\lambda_{i j k}^{\prime}\left(L_{i}^{0} D_{j} D_{k}^{c}-E_{i} V_{j l}^{\dagger} U_{l} D_{k}^{c}\right)+\frac{1}{2} \lambda_{i j k}^{\prime \prime} U_{i}^{c} D_{j}^{c} D_{k}^{c},
\end{aligned}
$$

where $L_{i}$ and $Q_{i}$ are the lepton and quark $S U(2)$ doublets, and $E_{i}^{c}, U_{i}^{c}, D_{i}^{c}$ are the $S U(2)$ singlet lepton and anti-quark superfields. Here $V_{i j}$ is the CKM matrix of quark fields, and the R-parity violating lepton-Higgsino mass mixing is described by dimensionless parameter $\epsilon_{i}$ in the unit of the conventional Higgsino mass $\mu$. From the above superpotential, one obtains the following lepton number violating Yukawa vertices in the Lagrangian;

$$
\begin{aligned}
-\mathcal{L}_{\mathbb{L}} & =\lambda_{i j k}\left(\tilde{\nu}_{i L} \bar{e}_{k R} e_{j L}+\tilde{e}_{j L} \bar{e}_{k R} \nu_{i L}+\tilde{e}_{k R}^{*} \bar{\nu}_{i R}^{C} e_{j L}\right) \\
& +\lambda_{i j k}^{\prime}\left(\tilde{\nu}_{i} \bar{d}_{k R} d_{j L}+\tilde{d}_{j L} \bar{d}_{k R} \nu_{i L}+\tilde{d}_{k R}^{*} \bar{\nu}_{i R}^{C} d_{j L}\right) \\
& -\lambda_{i l k}^{\prime} V_{l j}^{\dagger}\left(\tilde{e}_{i L} \bar{d}_{k R} u_{j L}+\tilde{u}_{j L} \bar{d}_{k R} e_{i L}+\tilde{d}_{k R}^{*} \bar{e}_{i R}^{C} u_{j L}\right)
\end{aligned}
$$

where the superscript $C$ denotes the charge conjugation. In order to ensure the longevity of proton, the products $\lambda^{\prime} \lambda^{\prime \prime}$ have to be highly suppressed [10]. Bilinear R-parity violating terms can also contribute to proton decay through the products $\left\langle\tilde{\nu}_{i}\right\rangle \lambda^{\prime \prime}$ or $\left(\epsilon_{i} \mu\right)^{*} \lambda^{\prime \prime}$ [11]. In this paper, we simply assume that $\lambda^{\prime \prime}$ is small enough to ensure the proton stability, while the L-violating couplings can take arbitrary values as long as they satisfy other phenomenological constraints.

So far we have defined the quark and lepton mass eigenbasis without including the effects of R-parity violation. In the presence of bilinear R-parity violation, there appear mixings between the ordinary fermions (Higgses) and the gauginos/Higgsinos (sleptons). As a result, further diagonalization is required to define the true mass eigenstates. In the below, we present the mixing matrices between the R-parity even and odd particles relevant for our calculation of EDMs. For this, we generalize the results in Ref. [12 to take into account the complexity of the bilinear parameters in Eqs. (2, 3, (3), which leads to a mixing between the CP even and CP odd neutral Higgses and sneutrinos. The process of diagonalization of slepton-Higgs mixing involves a rotation by which the Goldstone modes in the Higgsslepton sector are decoupled [12]. For the sake of simplicity, we go to the new basis in which the lepton and $Y=-1 / 2$ Higgs superfields are redefined so that sneutrinos have vanishing VEVs [13]. When the sneutrino VEVs in the original basis are small enough, i.e. $a_{i} \equiv\left\langle\tilde{\nu}_{i}^{*}\right\rangle /\left\langle H_{1}^{0}\right\rangle \ll 1$ in Eq. (4), the Lagrangian in the new basis can be obtained by the field redefinition 


$$
L_{i}=\hat{L}_{i}+a_{i}^{*} \hat{H}_{1}, \quad H_{1}=\hat{H}_{i}-a_{i} \hat{L}_{1}
$$

Then, the R-parity odd parameters in the new basis are given by

$$
\begin{aligned}
& \hat{B}_{i}=B_{i}-a_{i} B, \quad \hat{m}_{L_{i} H_{1}}^{2}=m_{L_{i} H_{1}}^{2}+a_{i}\left(m_{L_{i}}^{2}-m_{H_{1}}^{2}\right) \\
& \hat{\epsilon}_{i}=\epsilon_{i}-a_{i}, \quad \hat{\lambda}_{i j k}=\lambda_{i j k}-a_{i} h_{j}^{e} \delta_{j k}, \quad \hat{\lambda}_{i j k}^{\prime}=\lambda_{i j k}^{\prime}-a_{i} h_{j}^{d} \delta_{j k}
\end{aligned}
$$

where $h_{j}^{e}$ and $h_{j}^{d}$ are the Yukawa couplings of the charged leptons and down-type quarks, respectively. Note that we have the relation $\hat{B}_{i} t_{\beta}+\hat{m}_{L_{i} H_{1}}^{2}=0$ where $t_{\beta} \equiv \tan \beta=\left\langle H_{2}^{0}\right\rangle /\left\langle H_{1}^{0}\right\rangle$, which assures that sneutrino VEVs vanish in the new basis. In the following, we will drop hats to denote quantities in this new basis of vanishing sneutrino VEVs.

Neutrino-neutralino mixing: Let us first consider the well-known mixing among neutral fermions [6] for the completeness. In the basis of vanishing sneutrino VEVs, the mass mixing between the neutrinos $\left(\nu_{i}\right)$ and the neutralinos $N_{I}=\left(\tilde{B}, \tilde{W}_{3}, \tilde{H}_{1}^{0}, \tilde{H}_{2}^{0}\right)$ are described by the following $3 \times 4$ matrix:

$$
\left(\mathbf{M}_{\mathbf{D}}\right)_{i I}=\left(0,0,0,-\epsilon_{i} \mu\right)
$$

Combined with the usual $4 \times 4$ neutralino mass matrix $\mathbf{M}_{\mathbf{N}}$ of $N_{I}=\left(\tilde{B}, \tilde{W}_{3}, \tilde{H}_{1}^{0}, \tilde{H}_{2}^{0}\right)$, the above $3 \times 4$ mixing matrix $\mathbf{M}_{\mathbf{D}}$ forms a $7 \times 7$ mass matrix of the neutrinos and neutralinos. At leading order in the expansion in powers of small $\mathbf{M}_{\mathbf{D}}$, the $7 \times 7$ neutral fermion mass matrix can be diagonalized by the rotation:

$$
\mathcal{U}^{N} \equiv\left(\begin{array}{cc}
1 & \Theta^{N} \\
-\left(\Theta^{N}\right)^{T} & 1
\end{array}\right)\left(\begin{array}{cc}
U^{\nu} & 0 \\
0 & N
\end{array}\right)
$$

where $\Theta^{N} \equiv \mathbf{M}_{D} \mathbf{M}_{N}^{-1}, U^{\nu}$ is the $3 \times 3$ neutrino mixing matrix, and finally $N$ is the usual $4 \times 4$ diagonalization matrix of $\mathbf{M}_{\mathbf{N}}$ in the absence of $\mathrm{R}$-parity violation 14 . Here the mixing matrix $\Theta^{N}$ is given by [15]

$$
\Theta_{i I}^{N}=\epsilon_{i} c_{I}^{N} c_{\beta}+\epsilon_{i} \delta_{I 3}
$$

with

$$
c_{I}^{N}=\frac{M_{Z}}{F_{N}}\left(s_{W} \frac{M_{2}}{M_{\tilde{\gamma}}},-c_{W} \frac{M_{1}}{M_{\tilde{\gamma}}}, s_{\beta} \frac{M_{Z}}{\mu},-c_{\beta} \frac{M_{Z}}{\mu}\right),
$$

where $F_{N} \equiv M_{1} M_{2} / M_{\tilde{\gamma}}-M_{Z}^{2} s_{2 \beta} / \mu, M_{\tilde{\gamma}} \equiv c_{W}^{2} M_{1}+s_{W}^{2} M_{2}$ for the $S U(2) \times U(1)$ gaugino mass parameters $M_{1}, M_{2}, s_{W}=\sin \theta_{W}$ with $\theta_{W}$ being the weak mixing angle, and $s_{\beta}=\sin \beta$, etc. In the above, for the sake of simplicity, we assume that $M_{1}, M_{2}$, and $\mu$ are approximately real, which may be necessary also to avoid a too large neutron EDM.

Charged lepton-chargino mixing: R-parity violation leads also a mixing between the charged leptons and the charged gaugino/Higgsino. Following the steps similar to the case of neutral fermions, one finds that the $5 \times 5$ diagonalization matrices $\mathcal{U}^{L}, \mathcal{U}^{R}$ which would bring $\left(e_{i}, \tilde{W}^{-}, \tilde{H}_{1}^{-}\right)$and $\left(e_{i}^{c}, \tilde{W}^{+}, \tilde{H}_{2}{ }^{+}\right)$to the mass eigenstates $\left(e_{i}^{ \pm}, \chi_{1}^{ \pm}, \chi_{2}^{ \pm}\right)$are given by

$$
\mathcal{U}^{L, R}=\left(\begin{array}{cc}
1 & \Theta^{L, R} \\
-\Theta^{L, R \dagger} & 1
\end{array}\right)\left(\begin{array}{cc}
1 & 0 \\
0 & L, R
\end{array}\right)
$$


where

$$
\Theta_{i a}^{L}=\epsilon_{i} c_{a}^{L} c_{\beta}+\epsilon_{i} \delta_{a 2}, \quad \Theta_{i a}^{R}=\frac{m_{i}^{e}}{F_{C}} \epsilon_{i} c_{a}^{R} c_{\beta}
$$

with

$$
\begin{aligned}
c_{a}^{L} & =-\left(\sqrt{2} \frac{M_{W}}{F_{C}}, 2 s_{\beta} \frac{M_{W}^{2}}{\mu F_{C}}\right) \\
c_{a}^{R} & =-\left(\sqrt{2} \frac{M_{W}\left(\mu-M_{2} t_{\beta}\right)}{\mu F_{C}}, \frac{\left(M_{2}^{2}+2 M_{W}^{2} c_{\beta}^{2}\right)}{c_{\beta} \mu F_{C}}\right) .
\end{aligned}
$$

Here $F_{C} \equiv M_{2}+M_{W}^{2} s_{\beta} / \mu$, and $m_{i}^{e}$ denote the charged lepton mass. Note that $\Theta_{i a}^{R}$ is suppressed by the factor $m_{i}^{e} / F_{C}$ compared to $\Theta_{i a}^{L}$. In Eq. (12), the $2 \times 2$ matrices $L, R$ are the usual chargino mass diagonalization matrices in the absence of R-parity violation [14], and the index $a=1,2$ labels the chargino fields before the diagonalization by $L, R$.

Charged slepton-charged Higgs mixing: In the basis with vanishing sneutrinos VEVs, no mass mixing between the slepton and Higgses arises from the D-term potential. Then the mixing comes from the following part of the scalar potential:

$$
V \ni B_{i}\left(L_{i} H_{2}-t_{\beta} L_{i} H_{1}^{\dagger}\right)+\epsilon_{i} h_{i}^{e} \mu H_{2} H_{1}^{\dagger} E_{i}^{c *}+\text { h.c. },
$$

where we have used the relation $m_{L_{i} H_{1}}^{2}=-B_{i} t_{\beta}$ for vanishing sneutrino VEVs. Decoupling the Goldstone mode by setting $H_{1}^{-}=s_{\beta} H^{-}$and $H_{2}^{-}=c_{\beta} H^{-}$, we find that the mixing mass terms for the charged slepton and Higgs fields are given by

$$
V \ni-\left(B_{i} / c_{\beta}\right) \tilde{e}_{i L} H^{+}-\left(m_{i}^{e} \mu / c_{\beta}\right) \tilde{e}_{i R} H^{+}+h . c .
$$

where $\tilde{e}_{i L}$ and $\tilde{e}_{i R}$ are the left-handed and right-handed sleptons, respectively. Applying again the seesaw diagonalization as in the case of Eqs. (9,12), one finds the $8 \times 7$ rotation matrix $\mathcal{U}^{C}$ which brings the charged scalar fields $\left(\tilde{e}_{i L}, \tilde{e}_{i R}, H_{1}^{-}, H_{2}^{-}\right)$to the mass eigenstates $\left(\tilde{e}_{i 1}, \tilde{e}_{i 2}, H^{-}\right)$:

$$
\mathcal{U}^{C}=\left(\begin{array}{ccc}
1 & 0 & \eta_{1}^{i} \\
0 & 1 & \eta_{2}^{i} \\
-\eta_{1}^{i *} s_{\beta} & -\eta_{2}^{i *} s_{\beta} & s_{\beta} \\
-\eta_{1}^{i *} c_{\beta} & -\eta_{2}^{i *} c_{\beta} & c_{\beta}
\end{array}\right)
$$

where

$$
\begin{aligned}
\eta_{1}^{i} & =\frac{B_{i}\left(M_{R i}^{2}-m_{H^{-}}^{2}\right)-\epsilon_{i} m_{i}^{e} \mu M_{D i}^{2}}{c_{\beta}\left(m_{\tilde{e}_{i 1}}^{2}-m_{H^{-}}^{2}\right)\left(m_{\tilde{e}_{i 2}}^{2}-m_{H^{-}}^{2}\right)} \\
\eta_{2}^{i} & =\frac{\epsilon_{i} m_{i}^{e} \mu\left(M_{L i}^{2}-m_{H^{-}}^{2}\right)-B_{i} M_{D i}^{2}}{c_{\beta}\left(m_{\tilde{e}_{i 1}}^{2}-m_{H^{-}}^{2}\right)\left(m_{\tilde{e}_{i 2}}^{2}-m_{H^{-}}^{2}\right)}
\end{aligned}
$$

for $M_{L i}^{2}, M_{R i}^{2}$ and $M_{D i}^{2}$ being the left-left, right-right and left-right mass terms of slepton fields in the case of R-parity conservation, respectively. Here we have neglected the diagonalization 
of the slepton mass matrix which is assumed to be an approximate unit matrix for reasonable range of $\tan \beta$.

Sneutrino-neutral Higgs mixing: If we allow $m_{L_{i} H_{1}}^{2}$ and/or $B_{i}$ to be complex-valued, these $R$ parity violating parameters lead to a mixing not only between the R-parity even and odd states, but also between the CP even and odd states. Then the usual decomposition of slepton/Higgs sector into CP-even and CP-odd parts [13] cannot be applied any more. Again decoupling the Goldstone mode by setting $\operatorname{Im}\left(H_{1}^{0}\right)=s_{\beta} A^{0}$ and $\operatorname{Im}\left(H_{2}^{0}\right)=c_{\beta} A^{0}$, we find the mixing mass terms for $\left(\operatorname{Re}\left(\tilde{\nu}_{i}\right), \operatorname{Im}\left(\tilde{\nu}_{i}\right)\right)$ and $\left(\operatorname{Re}\left(H_{1}\right), \operatorname{Re}\left(H_{2}\right), A^{0}\right)$ are given by

$$
\left(\begin{array}{ccc}
-\operatorname{Re}\left(B_{i}\right) t_{\beta} & \operatorname{Re}\left(B_{i}\right) & -\operatorname{Im}\left(B_{i}\right) / c_{\beta} \\
\operatorname{Im}\left(B_{i}\right) t_{\beta} & -\operatorname{Im}\left(B_{i}\right) & -\operatorname{Re}\left(B_{i}\right) / c_{\beta}
\end{array}\right)
$$

Combining the above mixing mass matrix with the R-parity conserving slepton and Higgs mass matrix, we get the diagonalization matrix given as follows:

$$
\mathcal{U}^{S}=\left(\begin{array}{cc}
1 & \Theta_{i}^{S} \\
-\left(\Theta_{i}^{S}\right)^{T} & 1
\end{array}\right)\left(\begin{array}{cc}
1 & 0 \\
0 & U_{\alpha}
\end{array}\right)
$$

where the index $i$ labels each slepton generation and $U_{\alpha}$ is a block-diagonal $3 \times 3$ matrix in which the upper-left block is a $2 \times 2$ matrix diagonalizing the CP-even Higgs bosons in the absence of R-parity violation [14] and the lower-right block is identity. Here we keep using the standard notation for the Higgs-like scalar fields although they are not exact CP eigenstates. Again, the slepton diagonalization part is neglected. In Eq. (18), $\Theta^{S}$ (for each slepton flavor $i$ ) is a $2 \times 3$ matrix whose components are given by

$$
\begin{array}{cc}
\Theta_{11}^{S}=\frac{\left(m_{\tilde{\nu}_{i}}^{2}+M_{Z}^{2} c_{2 \beta}\right) t_{\beta} \operatorname{Re}\left(B_{i}\right)}{F_{S}}, & \Theta_{21}^{S}=-\frac{\left(m_{\tilde{\nu}_{i}}^{2}+M_{Z}^{2} c_{2 \beta}\right) t_{\beta} \operatorname{Im}\left(B_{i}\right)}{F_{S}} \\
\Theta_{12}^{S}=-\frac{\left(m_{\tilde{\nu}_{i}}^{2}-M_{Z}^{2} c_{2 \beta}\right) \operatorname{Re}\left(B_{i}\right)}{F_{S}}, & \Theta_{22}^{S}=\frac{\left(m_{\tilde{\nu}_{i}}^{2}-M_{Z}^{2} c_{2 \beta}\right) \operatorname{Im}\left(B_{i}\right)}{F_{S}} \\
\Theta_{13}^{S}=\frac{\operatorname{Im}\left(B_{i}\right)}{c_{\beta}\left(m_{\tilde{\nu}_{i}}^{2}-m_{A}^{2}\right)}, & \Theta_{23}^{S}=\frac{\operatorname{Re}\left(B_{i}\right)}{c_{\beta}\left(m_{\tilde{\nu}_{i}}^{2}-m_{A}^{2}\right)}
\end{array}
$$

where $F_{S} \equiv m_{\tilde{\nu}_{i}}^{2}\left(m_{\tilde{\nu}_{i}}^{2}-m_{h^{0}}^{2}-m_{H^{0}}^{2}\right)+m_{h^{0}}^{2} m_{H^{0}}^{2}$ and $m_{\tilde{\nu}_{i}}^{2}=m_{L_{i}}^{2}+M_{Z}^{2} c_{2 \beta} / 2$. As we will see in the Appendix, the EDMs arising from slepton-Higgs mixing turns out to be proportional to the charged lepton or down-type Yukawa couplings [see the terms containing $\mathcal{U}^{S}$ in Eqs. (A2, A3)] and thus are subleading.

An important consequence of neutrino-neutralino mixing is the generation of nonzero neutrino mass [6]. Diagonalizing the $7 \times 7$ neutral fermion mass matrix, one finds the neutrino mass matrix at tree-level:

$$
m_{i j}^{\mathrm{tree}}=\frac{M_{Z}^{2}}{F_{N}} c_{\beta}^{2} \epsilon_{i} \epsilon_{j}
$$

The existing atmospheric and solar neutrino data suggest that the largest neutrino mass eigenvalue is of the order of $0.1 \mathrm{eV}$ [16]. Also the neutrino masses induced by R-parity violation are generically hierarchical and dominated by the above tree level mass matrix. We then have 


$$
\epsilon \equiv\left(\sum_{i}\left|\epsilon_{i}\right|^{2}\right)^{1 / 2} \simeq 0.9 \times 10^{-6} \frac{1}{c_{\beta}}\left(\frac{F_{N}}{M_{Z}}\right)^{\frac{1}{2}}\left(\frac{m_{\nu_{3}}}{0.1 \mathrm{eV}}\right)^{\frac{1}{2}}
$$

where $m_{\nu_{3}}$ denotes the largest mass eigenvalue of (20). If $\mathrm{R}$-parity violation is the dominant source of neutrino masses, the large mixing of atmospheric neutrinos [16] would imply $\epsilon \approx$ $\left|\epsilon_{2}\right| \approx\left|\epsilon_{3}\right|$, and one can have $\left|\epsilon_{1}\right| \approx \epsilon$ or $\left|\epsilon_{1}\right| \approx 0.04 \epsilon$ depending on the choice of the large or small mixing angle solution to the solar neutrino problem [17.

\section{FERMION EDM FROM R-PARITY VIOLATION}

The fermion EDM operator is given by

$$
\frac{i}{2} d_{\psi} \bar{\psi} \sigma^{\mu \nu} \gamma_{5} \psi F_{\mu \nu}
$$

for a Dirac fermion $\psi$ and the electromagnetic field strength $F_{\mu \nu}$. Obviously this EDM operator flips the fermion chirality and also breaks CP invariance. It has been argued that there is no one-loop fermion EDM from R-parity violating trilinear Yukawa couplings in the limit of massless neutrino [7],8]. To generate R-parity even EDM, one needs to insert even number of R-parity odd couplings. Then it is not possible to have one-loop EDM diagrams involving the two insertions of R-parity violating Yukawa couplings in a manner compatible with both the requirement of chirality-flip and the chiral structure of Yukawa vertices. However in the presence of bilinear R-parity violation which would yield a mixing between the R-parity even and odd particles, a combination of R-parity violating bilinear and trilinear couplings can induce a fermion EDM at one-loop level. An important feature of these one-loop diagrams is that the second trilinear interaction vertex is provided by gauge interactions (or the large top quark Yukawa coupling), so there is no small fermion mass or Yukawa couplings involved other than the minimal product of R-parity odd bilinear and trilinear couplings. FIGs. 1-3 show such diagrams for the EDMs of charged lepton, down-type quarks, and up-type quarks, respectively. One can also have one-loop diagrams involving the two insertions of R-parity odd bilinear couplings. However such diagrams are suppressed by small neutrino mass, and turn out to be negligibly small.

Let us first consider the leading contributions to the electron EDM in more detail. The diagrams of FIG. 1 without a flavor change of sleptons inside the loop, i.e. $i=k=\alpha=1$, give rise to the electron EDM :

$$
\begin{aligned}
d_{e}=\frac{e g}{16 \pi^{2}} & \left\{\operatorname{Im}\left(\mathcal{U}_{W \chi_{n}}^{R} \mathcal{U}_{j \chi_{n}}^{L} \lambda_{1 j 1}\right) \frac{1}{m_{\tilde{\nu}_{1}}} G_{f}\left(m_{\chi_{n}^{ \pm}} ; m_{\tilde{\nu}_{1}}\right)\right. \\
& -\frac{1}{\sqrt{2}} \operatorname{Im}\left(\left[\mathcal{U}_{W \chi_{n}}^{N}+t_{W} \mathcal{U}_{B \chi_{n}}^{N}\right] \mathcal{U}_{j \chi_{n}}^{N} \lambda_{1 j 1}\right) \frac{1}{m_{\tilde{e}_{1}}} G_{s}\left(m_{\chi_{n}^{0}} ; m_{\tilde{e}_{1}}\right) \\
& \left.+\sqrt{2} t_{W} \operatorname{Im}\left(\mathcal{U}_{B \chi_{n}}^{N} \mathcal{U}_{j \chi_{n}}^{N} \lambda_{1 j 1}\right) \frac{1}{m_{\tilde{e}_{1}^{c}}} G_{s}\left(m_{\chi_{n}^{0}} ; m_{\tilde{e}_{1}^{c}}\right)\right\},
\end{aligned}
$$

where $G_{f}, G_{s}$ are the loop functions defined in the Appendix and $\mathcal{U}^{L}, \mathcal{U}^{R}, \mathcal{U}^{N}$ are the diagonalization matrices discussed in the previous section. Here $\chi_{n}^{ \pm}, \chi_{n}^{0}$ are the fermions inside 
the loops of FIG. 1 which can be either R-parity even, i.e. charged lepton or neutrino, or R-parity odd, i.e. chargino or neutralino. It turns out that the terms with R-parity even $\chi_{n}$ is negligible, so we will keep only the terms with R-parity odd $\chi_{n}$. In this case, $\mathcal{U}_{W \chi_{n}}^{R}$, $\mathcal{U}_{W \chi_{n}}^{N}, \mathcal{U}_{B \chi_{n}}^{N}$ correspond to the R-parity even elements of the usual chargino or neutralino diagonalization matrix, while $\mathcal{U}_{j \chi_{n}}^{L}$ or $\mathcal{U}_{j \chi_{n}}^{N}$ contains the R-parity odd element $\Theta_{j I}^{N}$ or $\Theta_{j a}^{L}$ which connects the neutrino with neutralino or the charged lepton with chargino.

Applying the experimental bound on the electron EDM to Eq. (23), we can obtain the bounds on the products of the trilinear and bilinear R-parity violating couplings. For a rough estimate, it is useful to recall that the mass mixing between the charged wino $\tilde{W}^{+}$ and the charged lepton $e_{j}$ (denoted by the cross in the fermion line of FIG. 1a) is given by $M_{W} \epsilon_{j} c_{\beta}$, implying

$$
\mathcal{U}_{W \chi_{n}}^{R} \mathcal{U}_{j \chi_{n}}^{L} m_{\chi_{n}^{ \pm}} \approx M_{W} \epsilon_{j} c_{\beta} .
$$

Then applying the experimental bound (11) to the first term of Eq. (23), we get

$$
\operatorname{Im}\left(\lambda_{1 j 1} \epsilon_{j}^{*} c_{\beta}\right), \operatorname{Im}\left(\lambda_{1 j 1} \epsilon_{j}^{*} \frac{M_{W}}{m_{\chi^{ \pm}}}\right)<1.4 \times 10^{-8} \frac{m_{\chi^{ \pm}} m_{\tilde{\nu}}}{(100 \mathrm{GeV})^{2}} .
$$

Here the bound on the product $\lambda_{1 j 1} \epsilon_{j}^{*} M_{W} / m_{\chi^{ \pm}}$can be understood by that the mixing mass of $\tilde{H}_{2}^{+}$and the charged lepton is given by $\epsilon_{j} \mu$ and the Wino-Higgsino mixing is of the order $M_{W} / m_{\chi}$ with $M_{W}<m_{\chi}$. For numerical estimates, we took a typical value of the loop functions, $G_{f}\left(m_{f} ; m_{\alpha}\right) \approx 1 / 3$ and $G_{s}\left(m_{f} ; m_{\alpha}\right) \approx 1 / 6$. Note that the values of these loop functions are rather insenstive to $m_{f} / m_{\alpha}$ as can be seen in FIG. 4 . The other terms in Eq. (23) are expected to yield a bit weaker bound by the factor of $t_{W}$. If the dominant source of neutrino masses were the bilinear R-parity violation, we have $\epsilon c_{\beta}=\left(F_{N} m_{\nu}\right)^{1 / 2} / M_{Z}$ as discussed in the previous section. This enables us to translate the bounds of Eq. (24) into a bound on the R-parity violating Yukawa coupling:

$$
\left|\lambda_{1 j 1}\right|<0.013 \frac{\epsilon}{\left|\epsilon_{j}\right|}\left(\frac{m_{\chi^{ \pm}}}{100 \mathrm{GeV}}\right)^{1 / 2}\left(\frac{m_{\tilde{\nu}}}{100 \mathrm{GeV}}\right)\left(\frac{m_{\nu}}{0.1 \mathrm{eV}}\right)^{-1 / 2},
$$

where we assumed that the phases are of order unity and $F_{N} \approx m_{\chi^{ \pm}}$.

If we allow a generation mixing in the squark or slepton sector, we have more contributions to the fermion dipole moments. To deal with this generation mixing, let us use the so-called mass insertion method [18]. In this approach, the amount of flavor violation is parameterized by the quantity,

$$
\delta_{i j}^{f, A B} \equiv \frac{\Delta_{i j}^{f, A B}}{m_{\tilde{f}_{i}} m_{\tilde{f}_{j}}},
$$

where $\Delta_{i j}^{f, A B}$ is the off-diagonal element and $m_{\tilde{f}_{i}}^{2}$ is the diagonal element of the sfermion mass-squared matrix in the quark and lepton mass eigenbasis, $f=u, d, l$ are the indices for the up-type squarks, down-type squarks, sleptons, and $A, B=L, R$ denote the chirality of these sfermions. Including the flavor mixing of sfermions, we have for instance the following contribution to the electron EDM: 


$$
d_{e}=\frac{e g}{16 \pi^{2}} \operatorname{Im}\left(\mathcal{U}_{W \chi_{n}}^{R} \mathcal{U}_{j \chi_{n}}^{L} \delta_{1 \alpha}^{l, L L} \lambda_{\alpha j 1}\right) H_{f}\left(m_{\chi_{n}^{ \pm}} ; m_{\tilde{\nu}_{1}}, m_{\tilde{\nu}_{\alpha}}\right)
$$

which reproduces the first term in Eq. (23) when $1=\alpha$. The definition of the loop function $H_{f}$ and also the other contributions can be found in the Appendix. Again applying the experimental bound to these contributions to the electron EDM, we get

$$
\begin{aligned}
& \operatorname{Im}\left(\delta_{1 \alpha}^{l, L L} \lambda_{\alpha j 1} \epsilon_{j}^{*} c_{\beta}\right), \operatorname{Im}\left(\delta_{1 \alpha}^{l, L L} \lambda_{\alpha j 1} \epsilon_{j}^{*} \frac{M_{W}}{m_{\chi}}\right)<1.4 \times 10^{-8} \frac{m_{\chi} m_{\tilde{l}}}{(100 \mathrm{GeV})^{2}} \\
& \operatorname{Im}\left(\delta_{1 \alpha}^{l, R R} \lambda_{1 j \alpha} \epsilon_{j}^{*} c_{\beta}\right), \operatorname{Im}\left(\delta_{1 \alpha}^{l, R R} \lambda_{1 j \alpha} \epsilon_{j}^{*} \frac{M_{W}}{m_{\chi}}\right)<6.4 \times 10^{-8} \frac{m_{\chi} m_{\tilde{l}}}{(100 \mathrm{GeV})^{2}},
\end{aligned}
$$

where the first bound comes from the diagram of FIG. 1a or 1b exchanging $\tilde{\nu}$ or $\tilde{e}$ and the second one from FIG. 1c exchanging $\tilde{e}^{c}$. Most stringent bounds on $\delta_{i j}^{l, A B}$ with $A B=L L, R R$ come from the $l_{i} \rightarrow l_{j} \gamma$ decays. One then finds $\delta_{12}^{l, A B} \lesssim 10^{-2}\left(m_{\tilde{l}} / 100 \mathrm{GeV}\right)^{2}$, however there are essentially no bounds on $\delta_{13}^{l, A B}$ and $\delta_{23}^{1, A B}$ [18].

The most important contributions to the down quark EDM come from the gauge vertex diagrams [FIG. 2a, 2b], and also the top Yukawa vertex diagrams [FIG. 2c], from which we find

$$
\begin{aligned}
d_{d}= & \frac{e g}{16 \pi^{2}}\left\{-\operatorname{Im}\left(\mathcal{U}_{j \chi_{n}^{-}}^{L} \mathcal{U}_{W \chi_{n}^{+}}^{R} V_{\alpha 1} V_{l \alpha}^{\dagger} \lambda_{j l 1}^{\prime}\right) \frac{1}{m_{\tilde{u}_{\alpha}}}\left[\frac{2}{3} G_{s}\left(m_{\chi_{n}}^{2} ; m_{\tilde{u}_{\alpha}}\right)+G_{f}\left(m_{\chi_{n}} ; m_{\tilde{u}_{\alpha}}\right)\right]\right. \\
& +\frac{1}{3 \sqrt{2}} \operatorname{Im}\left(\left[\mathcal{U}_{W \chi_{n}}^{N}-\frac{t_{W}}{3} \mathcal{U}_{B \chi_{n}}^{N}\right] \mathcal{U}_{j \chi_{n}^{0}}^{N} \lambda_{j 11}\right) \frac{1}{m_{\tilde{d}_{1}}} G_{s}\left(m_{\chi_{n}^{0}} ; m_{\tilde{d}_{1}}\right) \\
& \left.+\frac{\sqrt{2}}{9} t_{W} \operatorname{Im}\left(\mathcal{U}_{B \chi_{n}}^{N} \mathcal{U}_{j \chi_{n}}^{N} \lambda_{j 11}^{\prime}\right) \frac{1}{m_{\tilde{d}_{1}^{c}}} G_{s}\left(m_{\chi_{n}^{0}} ; m_{\tilde{d}^{c} 1}\right)\right\} \\
- & \frac{e h_{t}}{16 \pi^{2}} \operatorname{Im}\left(\mathcal{U}_{H_{2} \phi_{n}^{-}}^{C *} \mathcal{U}_{j \phi_{n}^{-}}^{C} V_{31} V_{l 3}^{\dagger} \lambda_{j l 1}^{\prime}\right) \frac{1}{m_{\phi_{n}^{ \pm}}}\left[\frac{2}{3} G_{f}\left(m_{t} ; m_{\phi_{n}^{ \pm}}\right)+G_{s}\left(m_{t} ; m_{\phi_{n}^{ \pm}}\right)\right] .
\end{aligned}
$$

More general expression including the squark mixing effects is given in the Appendix. From the valence quark contribution to the neutron EDM

$$
d_{n}=\frac{1}{3}\left(4 d_{d}-d_{u}\right) r^{e}
$$

taking into account the renormalization group effect from the weak scale to the hadronic scale with $r^{e} \simeq 1.5$ [19], the current experimental bound (11) gives

$$
\operatorname{Im}\left(V_{\alpha 1} V_{l \alpha}^{\dagger} \lambda_{j l 1}^{\prime} \epsilon_{j}^{*} c_{\beta},\right), \operatorname{Im}\left(V_{\alpha 1} V_{l \alpha}^{\dagger} \lambda_{j l 1}^{\prime} \epsilon_{j}^{*} \frac{M_{W}}{m_{\chi}}\right)<1.0 \times 10^{-7} \frac{m_{\chi} m_{\tilde{q}}}{(100 \mathrm{GeV})^{2}}
$$

Again if the bilinear R-parity violation gives $m_{\nu} \approx 0.1 \mathrm{eV}$, the above bound would give $\lambda_{j 11}^{\prime} \lesssim 0.1$. From the top quark exchange diagram [FIG. 2c] giving the last contribution of Eq. (29), one finds

$$
\mathcal{U}_{H_{2} \phi_{n}^{-}}^{C *} \mathcal{U}_{j \phi_{n}^{-}}^{C} \approx \eta_{1}^{j *} c_{\beta}
$$


as can be read off from Eq. (16). The contribution involving $\eta_{2}^{i}$ is neglected as it is proportional to the lepton mass $m_{e}^{i}$. Then one gets

$$
\operatorname{Im}\left(V_{l 3}^{\dagger} \lambda_{j l 1}^{\prime} \frac{B_{j}^{*}}{m_{\tilde{l}}^{2}}\right)<2.5 \times 10^{-6} \frac{0.01}{V_{31}} \frac{m_{\tilde{l}}}{100 \mathrm{GeV}}
$$

If we allow the squark generation mixing, $V_{\alpha 1} V_{l \alpha}^{\dagger} \lambda_{j l 1}^{\prime}$ can be replaced by $\lambda_{i \alpha 1}^{\prime} \delta_{\alpha 1}^{U, L L}$ in Eq. (30). More general expressions with both the CKM and squark mixings are given in the Appendix.

Contrary to the down quark EDM, the up quark EDM in our scheme turns out to be very small as it is further suppresed by small quark masses. For instance, the diagram in FIG. 3 gives rise to

$$
d_{u}=\frac{e h_{u}}{16 \pi^{2}} \operatorname{Im}\left(\mathcal{U}_{i \chi_{n}}^{L} \mathcal{U}_{H \chi_{n}}^{R} V_{1 \alpha} V_{\beta 1}^{\dagger} \delta_{\alpha \alpha}^{d, L R} \lambda_{i \beta \alpha}^{\prime}\right) \frac{1}{m_{\tilde{d}_{\alpha}}}\left[-\frac{1}{3} G_{s}\left(m_{\chi_{n}^{-}} ; m_{\tilde{d}_{\alpha}}\right)+G_{f}\left(m_{\chi_{n}^{-}} ; m_{\tilde{d}_{\alpha}}\right)\right]
$$

which is the limiting case of $\alpha=\beta$ in Eq. (A4). One can see from Eq. (32) that the up quark EDM includes additional suppression factor $m_{u} m_{d} / m_{\tilde{q}}^{2}$.

The nucleon EDM gets contributions not only from the quark EDMs, but also from the chromoelectric dipole moments (CDMs) of light quarks [20]. Since the diagrams for the CDMs of the up and down quarks have the same structure as those for the EDMs under the replacement of the external photon by gluons, one does not find a new bound on the R-parity violating couplings. On the other hand, there is also a contribution from the strange quark CDM whose diagrams involve a different set of R-parity violating couplings. The strange quark CDM $\left(d_{s}^{c}\right)$ contribution to the neutron EDM is given by $d_{n} \approx 0.027 d_{s}^{c} r^{c}$ 221 with $r^{c} \simeq 3.3$ coming from the renormalization group effect, which leads to

$$
\operatorname{Im}\left(V_{\alpha 2} V_{l \alpha}^{\dagger} \lambda_{j l 2}^{\prime} \epsilon_{j}^{*} c_{\beta}\right), \operatorname{Im}\left(V_{\alpha 2} V_{l \alpha}^{\dagger} \lambda_{j l 2}^{\prime} \epsilon_{j}^{*} \frac{M_{W}}{m_{\chi}}\right)<1.7 \times 10^{-6} \frac{m_{\chi} m_{\tilde{q}}}{(100 \mathrm{GeV})^{2}}
$$

Collecting all the contributions to the electron and neutron EDMs, we summarize the resulting bounds on R-parity violating couplings in the Tables.

\section{CONCLUSION}

We have analyzed the electron and neutron EDMs induced by R-parity violating interactions, taking into account both the bilinear and trilinear R-parity violating terms. It is pointed out that the fermion EDMs can arise at one-loop level as a combined effect of the bilinear and trilinear terms. This has to be contrasted to the previous analyses showing that nonzero EDMs arise at two-loop level considering only the trilinear R-parity violating vertices. We have computed the complete one-loop diagrams (except those proportional to tiny neutrino masses) involving both the bilinear and trilinear parameters as presented in the Appendix. Among them, the leading contribution comes from the diagrams with a gauge/top-Yukawa vertex and a R-parity violating trilinear vertex for which the chiralityflip is provided by (bilinear) mass mixing between R-parity even and odd particles. These 
one-loop diagrams lead to somewhat severe constraints on the products of the R-parity violating bilinear and trilinear couplings as summarized in Table I. If the bilinear R-parity violation is a dominant source for the neutrino mass $m_{\nu} \sim 0.1 \mathrm{eV}$ as was suggested by the Super-Kamionkande atmospheric neutrino data, the typical upper bounds of $\mathcal{O}\left(10^{-2}\right)$ can be put on some $\lambda_{i j k}$ couplings involving the first generation index as shown in Table II.

Acknowledgement This work is supported by Seoam Foundation (K.C.) and by grant No. 1999-2-111-002-5 from the interdisciplinary Research program of the KOSEF and BK21 project of the Ministry of Education (K.C. and K.H). 


\section{REFERENCES}

[1] K. Abdullah et al., Phys. Rev. Lett. 65, 2340 (1990); E. Commins et al., Phys. Rev. A 50 (1994) 2960; B.E. Sauer, J. Wang and E.A. Hinds, Phys. Rev. Lett. 74, 1554 (1995); J. Chem. Phys 105 (1996) 7412.

[2] P.G. Harris et al., Phys. Rev. Lett. 82, 904 (1999).

[3] J. Ellis, S. Ferrara and D.V. Nanopoulos, Phys. Lett. B114, 231 (1982); W. Buchmuller and D. Wyler, Phys. Lett. B121, 321 (1983); J. Polchinski and M.B. Wise, Phys. Lett. B125, 393 (1983); J-M. Gerard, et al., Nucl. Phys. B253, 93 (1985); M. Dugan, B. Greenstein and L. Hall, Nucl. Phys. B255, 413 (1985); A. Sanda, Phys. Rev. D32, $2992(1985)$.

[4] T. Ibrahim and P. Nath, Phys. Lett. B418, 98 (1998); Phys. Rev. D57, 478 (1998); T. Falk and K.A. Olive, Phys. Lett. B439, 71 (1998); M. Brhlik, G.J. Good and G.L. Kane, Phys. Rev. D59, 115004 (1999); S. Pokorski, J. Rosiek and C.A. Savoy, Nucl. Phys. B570, 81 (2000).

[5] V. Ben-Hamo and Y. Nir, Phys. Lett. B339, 77 (1994); T. Banks, et al., Phys. Rev. D52, 5319 (1995); E. J. Chun and A. Lukas, Phys. Lett. B387, 99 (1996); K. Choi, E. J. Chun and H. Kim, Phys. Rev. D55, 7010 (1997); K. Choi, E. J. Chun and H. Kim, Phys. Lett. B394, 89 (1997); G. Eyal and Y. Nir, JHEP 9906, 025 (1999); Q. Shafi and Z. Tavartkiladze, hep-ph/9909238.

[6] L. Hall and M. Suzuki, Nucl. Phys. B231, 419 (1984); F. M. Borzumati, et al., Phys. Lett. B384 (1996) 123; B. de Carlos and P. L. White, Phys. Rev. D54 (1996) 3427; A. Yu. Smirnov and F. Vissani, Nucl. Phys. B460 (1996) 37; R. Hempfling, Nucl. Phys B478 (1996) 3; H. P. Nilles and N. Polonsky, Nucl. Phys. B484 (1997) 33; E. Nardi, Phys. Rev. D55 (1997) 5772; E. J. Chun, et al., Nucl. Phys. B544, 89 (1999); A. S. Joshipura and S. K. Vempati, Phys. Rev. D60, 095009 (1999); J. Ferrandis, Phys. Rev. D60, 095012 (1999); M. Bisset, et al., hep-ph/9811498; S. Rakshit, G. Bhattacharyya, Phys. Rev. D59, 091701 (1999); R. Adhikari, G. Omanovic, Phys. Rev. D59, 073003 (1999); K. Choi, E. J. Chun and K. Hwang, Phys. Rev. D60, 031301 (1999); D. E. Kaplan and A. Nelson, hep-ph/9901254; A. Abada and M. Losada, hep-ph/9908352; J. L. Chkareuli, et al., hep-ph/9908451; J. C. Romao, et al., hep-ph/9907499; O. Haug, et al., hep-ph/9909318.

[7] R.M. Godbole, S. Pakvasa, S.D. Rindani and X. Tata, hep-ph/9912315

[8] S.A. Abel, A. Dedes and H.K. Dreiner, hep-ph/9912429

[9] S.M. Barr, E.M. Friere and A. Zee, Phys. Rev. Lett. 65, 2626 (1990).

[10] I. Hinchliffe and T. Kaeding, Phys. Rev. D47, 279 (1993); A.Y. Simrnov and F. Vissani, Phys. Lett. B380, 317 (1996).

[11] B. Mukhopadhyaya, et al., Phys. Lett. B443, 191 (1998); E.J. Chun and J.S. Lee, Phys. Rev. D60, 075006 (1999).

[12] E.J. Chun and S.K. Kang, Phys. Rev. D61, 078012 (2000), hep-ph/9909429.

[13] Y. Grossman and H.E. Haber, Phys. Rev. D59, 093008 (1999).

[14] For a review, see H. Haber and G. Kane, Phys. Rep. 117, 75 (1985).

[15] A.S. Joshipura and M. Nowakowski, Phys. Rev. D51, 2421 (1995); M. Nowakowski and A. Pilaftsis, Nucl. Phys. B461, 19 (1996). 
[16] The Super-Kamiokande Collaboration, Y. Fukuda, et al., Phys. Rev. Lett. 81, 1562 (1998).

[17] For recent analyses, see, J.N. Bahcall, P.I. Krastev and A.Y. Smirnov, Phys. Rev. D58, 096016 (1998).

[18] F. Gabbiani, et al., Nucl. Phys. B477, 321 (1996); M. Misiak, S. Pokorski and J. Rosiek, in "Heavy Flavours II", ed A.J. Buras and M. Lindner (World Scientific, 1998), hep-ph/9703442.

[19] E. Braaten, C.S. Li and T.C. Yuan, Phys. Rev. Lett. 64, 1709 (1990); R. Arnowitt, J. Lopez and D.V. Nanopoulos, Phys. Rev. D42, 2423 (1990).

[20] X.G. He, B. McKeller and S. Pakvasa, Int. J. Mod. Phys. A4, 5011 (1989); Erratum, ibid. A6,1063 (1991)

[21] X.G. He, B. McKeller and S. Pakvasa, Phys. Lett. B254, 231 (1991). 


\section{TABLES}

\begin{tabular}{|c|c|}
\hline \hline coupling & upper bound \\
\hline$\lambda_{1 j 1} \epsilon_{j}^{*} c_{\beta}$ & $1.4 \times 10^{-8} x_{s}$ \\
$\delta_{13}^{l, L L} \lambda_{231} \epsilon_{2}^{*} c_{\beta}$ & $1.4 \times 10^{-8} x_{s}$ \\
$\delta_{12}^{l, L L} \lambda_{1 j 2} \epsilon_{j}^{*} c_{\beta}$ & $0.64 \times 10^{-7} x_{s}$ \\
$\delta_{13}^{l, L L} \lambda_{1 j 3} \epsilon_{j}^{*} c_{\beta}$ & $0.64 \times 10^{-7} x_{s}$ \\
\hline$V_{l \alpha}^{\dagger} V_{\alpha 1} \lambda_{j l 1}^{\prime} \epsilon_{j}^{*} c_{\beta}$ & $1.0 \times 10^{-7} x_{s}$ \\
$\delta_{1 l}^{l, L L} \lambda_{j l 1}^{\prime} \epsilon_{j}^{*} c_{\beta}$ & $1.0 \times 10^{-7} x_{s}$ \\
$V_{l \alpha}^{\dagger} V_{\alpha 2} \lambda_{j l 2}^{\prime} \epsilon_{j}^{*} c_{\beta}$ & $1.7 \times 10^{-6} x_{s}$ \\
$V_{l 3}^{\dagger} \lambda_{j l 1}^{\prime} \frac{B_{j}^{*}}{m_{\tilde{l}}^{2}}$ & $2.5 \times 10^{-6}\left(\frac{0.01}{V_{31}} \frac{m_{\tilde{l}}}{100 \mathrm{GeV}}\right)$ \\
\hline \hline
\end{tabular}

TABLE I. Bounds on the imaginart part of the combinations of trilinear and bilinear couplings. Note that $\epsilon_{j}^{*} c_{\beta}$ can be replaced by $\epsilon_{j}^{*} M_{W} / m_{\chi}$. Here $x_{s} \equiv \frac{m_{\chi} m_{\tilde{f}}}{\left(100 \mathrm{GeV}^{2}\right)}$.

\begin{tabular}{|c|c|}
\hline \hline coupling & upper bound \\
\hline$\lambda_{121}, \lambda_{131}$ & $0.013 x_{s} x_{\nu}$ \\
$\delta_{13}^{l, L L} \lambda_{231}$ & $0.013 x_{s} x_{\nu}$ \\
$\delta_{13}^{l, L L} \lambda_{123}$ & $0.059 x_{s} x_{\nu}$ \\
$\delta_{13}^{l, L} \lambda_{133}$ & $0.059 x_{s} x_{\nu}$ \\
\hline$\lambda_{j 11}^{\prime}$ & $0.10 x_{s} x_{\nu}$ \\
$\lambda_{j 21}^{\prime}$ & $0.45 x_{s} x_{\nu}$ \\
$\delta_{13}^{u, L L} \lambda_{j 31}^{\prime}$ & $0.10 x_{s} x_{\nu}$ \\
\hline \hline
\end{tabular}

TABLE II. Bounds on the magnitude of single trilinear coupling under the assumption that neutrino masses come dominantly from the R-parity violating bilinear terms. As a reference, we take $m_{\nu} \sim 0.1 \mathrm{eV}$ and then $x_{\nu} \equiv\left(\frac{F_{N}}{100 \mathrm{GeV}}\right)^{-1}\left(\frac{m_{\nu}}{0.1 \mathrm{eV}}\right)^{-\frac{1}{2}}$. 


\section{APPENDIX A: FULL ONE-LOOP CONTRIBUTIONS TO THE FERMION DIPOLE MOMENTS FROM R-PARITY VIOLATION}

In this Appendix, we provide complete expressions for the one-loop fermion dipole moments induced by R-parity violating couplings. For this, let us first define some loop functions. For the basic loop functions given by

$$
\begin{aligned}
G_{f}(t) & \equiv \frac{t-3}{2(1-t)^{2}}-\frac{\ln t}{(1-t)^{3}}, \\
G_{s}(t) & \equiv \frac{t+1}{2(1-t)^{2}}+\frac{t \ln t}{(1-t)^{3}},
\end{aligned}
$$

the modified loop functions which are propotional to the chirality-flipping fermion masses are defined as

$$
\begin{aligned}
G_{f}\left(m_{f} ; m_{\alpha}\right) & \equiv \frac{m_{f}}{m_{\alpha}} G_{f}\left(\frac{m_{f}^{2}}{m_{\alpha}^{2}}\right), \\
G_{s}\left(m_{f} ; m_{\alpha}\right) & \equiv \frac{m_{f}}{m_{\alpha}} G_{s}\left(\frac{m_{f}^{2}}{m_{\alpha}^{2}}\right) .
\end{aligned}
$$

These functions behave well in the limit $m_{f} \rightarrow 0$ and mildly depend on $m_{f} / m_{\alpha}$ as shown in FIG. 4. When the mass insertion approximation is used, another useful set of (dimensionful) loop functions are

$$
\begin{aligned}
& H_{f}\left(m_{f} ; m_{\alpha}, m_{\beta}\right)=\frac{m_{f} m_{\alpha} m_{\beta}}{m_{\alpha}^{2}-m_{\beta}^{2}}\left[\frac{1}{m_{\alpha}^{2}} G_{f}\left(\frac{m_{f}^{2}}{m_{\alpha}^{2}}\right)-\frac{1}{m_{\beta}^{2}} G_{f}\left(\frac{m_{f}^{2}}{m_{\beta}^{2}}\right)\right] \\
& H_{s}\left(m_{f} ; m_{\alpha}, m_{\beta}\right)=\frac{m_{f} m_{\alpha} m_{\beta}}{m_{\alpha}^{2}-m_{\beta}^{2}}\left[\frac{1}{m_{\alpha}^{2}} G_{s}\left(\frac{m_{f}^{2}}{m_{\alpha}^{2}}\right)-\frac{1}{m_{\beta}^{2}} G_{s}\left(\frac{m_{f}^{2}}{m_{\beta}^{2}}\right)\right]
\end{aligned}
$$

Any contribution to the electric dipole moment between the two fermions $f_{i}$ and $f_{k}$ can be expressed in the following form:

$$
d_{f, i k}=\frac{e}{16 \pi^{2}} \frac{1}{2 i}\left[\left(\delta_{\alpha \beta}^{f, A B} \cdot \mathcal{A}\right)_{i k}-\left(\delta_{\alpha \beta}^{f, A B} \cdot \mathcal{A}\right)_{k i}^{*}\right]
$$

where $f$ stands for the external quarks or charged leptons, $i, k$ are their generation indices, $A B$ denote the chirality of intermediate sfermions, i.e. $A B=L L, L R, R R$, and $\alpha$ and $\beta$ are the indices for those sfermions. Here we do not seperately treat the diagrams without sfermion flavor mixing. The expressions for such diagrams can be easily obtained from those for the diagrams with sfermion flavor mixing by changing $\delta_{\alpha \beta}^{f, A B}, H_{f}, H_{s}$ as follows:

$$
\begin{aligned}
\delta_{\alpha \beta}^{f, A B} & \rightarrow \delta_{\alpha \beta}, \\
H_{f}\left(m_{f} ; m_{\alpha}, m_{\beta}\right) & \rightarrow \frac{1}{m_{\alpha}} G_{f}\left(m_{f} ; m_{\alpha}\right), \\
H_{s}\left(m_{f} ; m_{\alpha}, m_{\beta}\right) & \rightarrow \frac{1}{m_{\alpha}} G_{s}\left(m_{f} ; m_{\alpha}\right) .
\end{aligned}
$$



by

The part $(\delta \cdot \mathcal{A})_{i k}$ for the charged lepton dipole moments between $l_{i}$ and $l_{k}$ are then given

$$
\begin{aligned}
(\delta \cdot \mathcal{A})_{i k}^{e}= & +g \mathcal{U}_{W \chi_{n}}^{R} \mathcal{U}_{j \chi_{n}}^{L} \delta_{i \alpha}^{l, L L} \lambda_{\alpha j k} H_{f}\left(m_{\chi_{n}^{ \pm}} ; m_{\tilde{\nu}_{i}}, m_{\tilde{\nu}_{\alpha}}\right) \\
& -\frac{1}{\sqrt{2}}\left(g \mathcal{U}_{W \chi_{n}}^{N}+g^{\prime} \mathcal{U}_{B \chi_{n}}^{N}\right) \mathcal{U}_{j \chi_{n}}^{N} \delta_{i \alpha}^{l, L L} \lambda_{\alpha j k} H_{s}\left(m_{\chi_{n}^{0}} ; m_{\tilde{e}_{i}}, m_{\tilde{e}_{\alpha}}\right) \\
& +\sqrt{2} g^{\prime} \mathcal{U}_{B \chi_{n}}^{N} \mathcal{U}_{j \chi_{n}}^{N} \delta_{k \alpha}^{l, R R} \lambda_{i j \alpha} H_{s}\left(m_{\chi_{n}^{0}} ; m_{\tilde{e}^{c}}, m_{\tilde{e}_{\alpha}}\right) \\
& +g \mathcal{U}_{W k}^{R} \delta_{l \alpha}^{l, L L} \lambda_{\alpha i l} H_{f}\left(m_{e_{l}} ; m_{\tilde{\nu}_{l}}, m_{\tilde{\nu}_{\alpha}}\right) \\
& -\frac{1}{2}\left[h_{i}^{e}\left\{\left(\mathcal{U}_{H_{1}^{R} \phi_{l}^{s}}^{S}+i \mathcal{U}_{H_{1}^{I} \phi_{l}^{s}}^{S}\right)\left(\mathcal{U}_{j^{R} \phi_{l}^{s}}^{S}+i \mathcal{U}_{j^{I} \phi_{l}^{s}}^{S}\right) \frac{1}{m_{\phi_{l}^{s}}} G_{f}\left(m_{e_{i}} ; m_{\phi_{l}^{s}}\right)\right\}+(i \leftrightarrow k)\right] \lambda_{j i k} \\
& +h_{i}^{e} \mathcal{U}_{H_{1} \chi_{n}}^{N} \mathcal{U}_{j \chi_{n}}^{N} \delta_{\alpha i}^{l, L R} \lambda_{j \alpha k} H_{s}\left(m_{\chi_{n}^{0}} ; m_{\tilde{e}_{\alpha}}, m_{\tilde{e}^{c_{i}}}\right) \\
& +h_{k}^{e} \mathcal{U}_{H_{1} \chi_{n}}^{N} \mathcal{U}_{j \chi_{n}}^{N} \delta_{\alpha k}^{l, L R} \lambda_{j i \alpha} H_{s}\left(m_{\chi_{n}^{0}} ; m_{\tilde{e}_{\alpha}}, m_{\tilde{e}_{i}}\right) \\
& -g V_{\alpha l} V_{\gamma \beta}^{\dagger} \mathcal{U}_{W k}^{R} \delta_{\alpha \beta}^{u, L L} \lambda_{i \gamma l}^{\prime}\left[2 H_{s}\left(m_{d_{l}} ; m_{\tilde{u}_{\alpha}}, m_{\tilde{u}_{\beta}}\right)+H_{f}\left(m_{d_{l}} ; m_{\tilde{u}_{\alpha}}, m_{\tilde{u}_{\beta}}\right)\right] \\
& +h_{\alpha}^{u} V_{\alpha l} V_{\gamma \beta}^{\dagger} \mathcal{U}_{H k}^{R} \delta_{\alpha \beta}^{u, R L} \lambda_{i \gamma l}^{\prime}\left[2 H_{s}\left(m_{d_{l}} ; m_{\tilde{u}_{\alpha}^{c}}, m_{\tilde{u}_{\beta}}\right)+H_{f}\left(m_{d_{l}} ; m_{\tilde{u}_{\alpha}}, m_{\tilde{u}_{\beta}}\right)\right] \\
& +h_{l}^{u} V_{l \alpha} V_{\gamma l}^{\dagger} \mathcal{U}_{H k}^{R} \delta_{\beta \alpha}^{d, R L} \lambda_{i \gamma \beta}^{\prime}\left[2 H_{s}\left(m_{u_{l}} ; m_{\tilde{d}^{c}}, m_{\tilde{d}_{\alpha}}\right)+H_{f}\left(m_{u_{l}} ; m_{\tilde{d}^{c} \gamma}, m_{\tilde{d}_{\alpha}}\right)\right]
\end{aligned}
$$

and for the dipole moments between the down type-quarks $d_{i}$ and $d_{k}$,

$$
\begin{aligned}
(\delta \cdot \mathcal{A})_{i k}^{d}= & -g \mathcal{U}_{j \chi_{n}^{-}}^{L} \mathcal{U}_{W \chi_{n}^{+}}^{R} V_{\alpha i} V_{l \beta}^{\dagger} \delta_{\alpha \beta}^{u, L L} \lambda_{j l k}^{\prime}\left[\frac{2}{3} H_{s}\left(m_{\chi_{n}} ; m_{\tilde{u}_{\alpha}}, m_{\tilde{u}_{\beta}}\right)+H_{f}\left(m_{\chi_{n}} ; m_{\tilde{u}_{\alpha}}, m_{\tilde{u}_{\beta}}\right)\right] \quad(\mathrm{A} 3) \\
& +\frac{1}{3 \sqrt{2}}\left(g \mathcal{U}_{W \chi_{n}}^{N}-\frac{g^{\prime}}{3} \mathcal{U}_{B \chi_{n}}^{N}\right) \mathcal{U}_{j \chi_{n}^{0}}^{N} \delta_{i \alpha}^{d, L L} \lambda_{j \alpha k} H_{s}\left(m_{\chi_{n}^{0}} ; m_{\tilde{d}_{i}}, m_{\tilde{d}_{\alpha}}\right) \\
& +\frac{\sqrt{2}}{9} g^{\prime} \mathcal{U}_{B \chi_{n}}^{N} \mathcal{U}_{j \chi_{n}}^{N} \delta_{k \alpha}^{d, R R} \lambda_{j i \alpha}^{\prime} H_{s}\left(m_{\chi_{n}^{0}} ; m_{\tilde{d}^{c}}, m_{\tilde{e}^{c} \alpha}\right) \\
& -h_{\alpha}^{u} \mathcal{U}_{H_{2} \phi_{n}^{-}}^{C *} \mathcal{U}_{j \phi_{n}^{-}}^{C} V_{\alpha i} V_{l \alpha}^{\dagger} \lambda_{j l k}^{\prime} \frac{1}{m_{\phi_{n}^{-}}}\left[\frac{2}{3} G_{f}\left(m_{u_{\alpha}} ; m_{\phi_{n}^{-}}\right)+G_{s}\left(m_{u_{\alpha}} ; m_{\phi_{n}^{-}}\right)\right] \\
& +h_{\alpha}^{u} \mathcal{U}_{j \chi_{n}^{-}}^{L} \mathcal{U}_{W \chi_{n}^{+}}^{R} V_{\alpha i} V_{l \beta}^{\dagger} \delta_{\beta \alpha}^{u, L R} \lambda_{j l k}^{\prime}\left[\frac{2}{3} H_{s}\left(m_{\chi_{n}} ; m_{\tilde{u}_{\beta}}, m_{\tilde{u}_{\alpha} \tilde{u}_{\alpha}}\right)+H_{f}\left(m_{\chi_{n}} ; m_{\tilde{u}_{\beta}}, m_{\tilde{u}_{\alpha}}\right)\right] \\
& -\frac{1}{6}\left[h_{i}^{d}\left\{\left(\mathcal{U}_{H_{1}^{R} \phi_{l}^{s}}^{S}+i \mathcal{U}_{H_{1}^{I} \phi_{l}^{s}}^{S}\right)\left(\mathcal{U}_{j^{R} \phi_{l}^{s}}^{S}+i \mathcal{U}_{j^{I} \phi_{l}^{s}}^{S}\right) \frac{1}{m_{\phi_{l}^{s}}} G_{f}\left(m_{d_{i}} ; m_{\phi_{l}^{s}}\right)\right\}+(i \leftrightarrow k)\right] \lambda_{j i k}^{\prime} \\
& +\frac{1}{3} h_{i}^{d} \mathcal{U}_{H_{1} \chi_{n}}^{N} \mathcal{U}_{j \chi_{n}}^{N} \delta_{\alpha j}^{d, L R} \lambda_{j \alpha k} H_{s}\left(m_{\chi_{n}^{0}} ; m_{\tilde{d}_{\alpha}}, m_{\tilde{d}^{c_{i}}}\right) \\
& +\frac{1}{3} h_{k}^{d} \mathcal{U}_{H_{1} \chi_{n}}^{N} \mathcal{U}_{j \chi_{n}}^{N} \delta_{\alpha k}^{d, R L} \lambda_{j i \alpha} H_{s}\left(m_{\chi_{n}^{0}} ; m_{\tilde{d}^{c} \alpha}, m_{\tilde{d}_{k}}\right),
\end{aligned}
$$

and finally for the up type quarks $u_{i}$ and $u_{k}$,

$$
\begin{aligned}
(\delta \cdot \mathcal{A})_{i k}^{u}= & -h_{k}^{u} \mathcal{U}_{H_{2} \phi_{n}^{-}}^{C *} \mathcal{U}_{j \phi_{n}^{-}}^{C} V_{k l} V_{\alpha i}^{\dagger} \lambda_{i \alpha l}^{\prime} \frac{1}{m_{\phi_{n}^{-}}}\left\{-\frac{1}{3} G_{f}\left(m_{d_{l}} ; m_{\phi_{n}^{-}}\right)+G_{s}\left(m_{d_{l}} ; m_{\phi_{n}^{-}}\right)\right\} \\
& -h_{k}^{u} \mathcal{U}_{j \chi_{n}}^{L} \mathcal{U}_{H_{\chi_{n}}}^{R} V_{k \alpha} V_{\gamma i}^{\dagger} \delta_{\alpha \beta}^{d, L R} \lambda_{j \gamma \beta}^{\prime}\left\{\frac{1}{3} H_{s}\left(m_{\chi_{n}^{ \pm}} ; m_{\tilde{d}_{\alpha}}, m_{\tilde{d}_{\beta}}\right)-H_{f}\left(m_{\chi_{n}^{ \pm}} ; m_{\tilde{d}_{\alpha}}, m_{\tilde{d}_{\beta}}\right)\right\}
\end{aligned}
$$

Here $\phi_{n}^{-}, \phi_{l}^{s}$ denotes the charged and neutral scalar mass-eigenstates, respectively. 


\section{FIGURES}

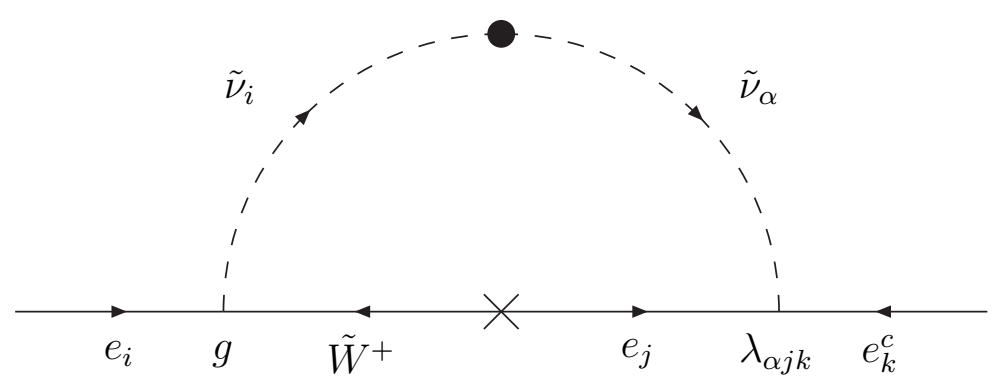

(a)

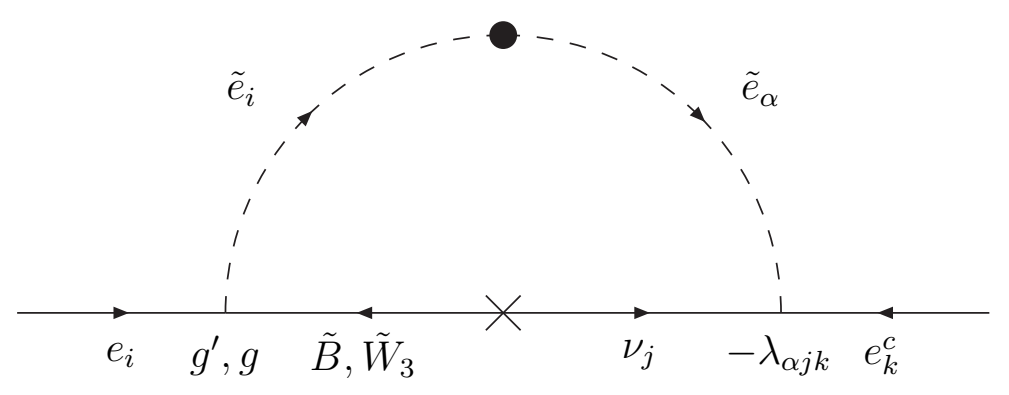

(b)

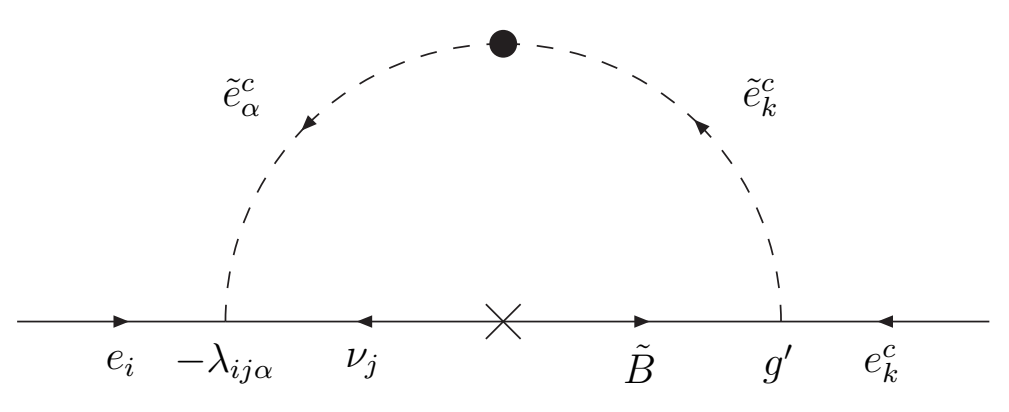

(c)

FIG. 1. Most important diagrams for the charged lepton EDM. The black blobs denote possible scalar mixing and the crosses are chirality-fliping mass insertions. 


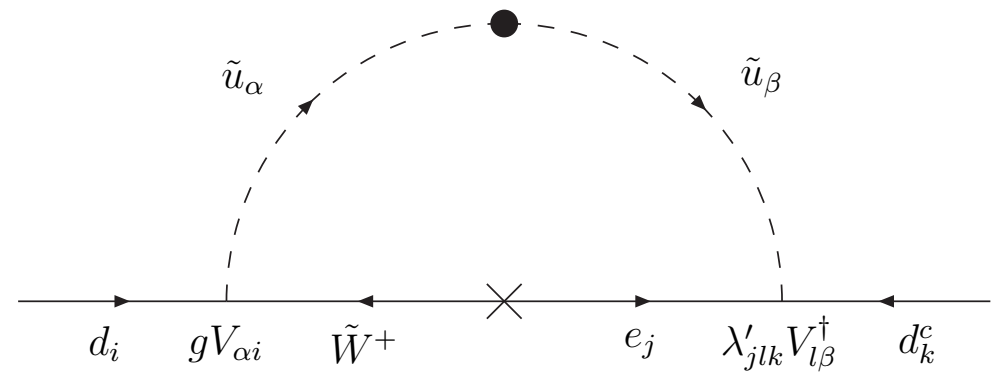

(a)

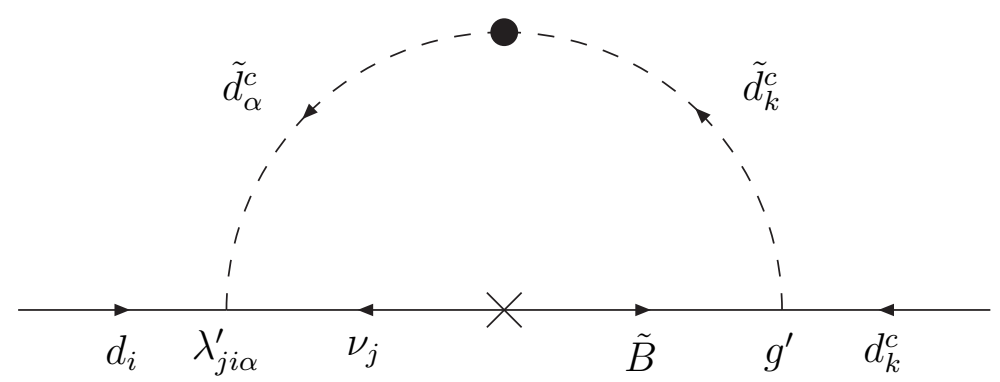

(b)

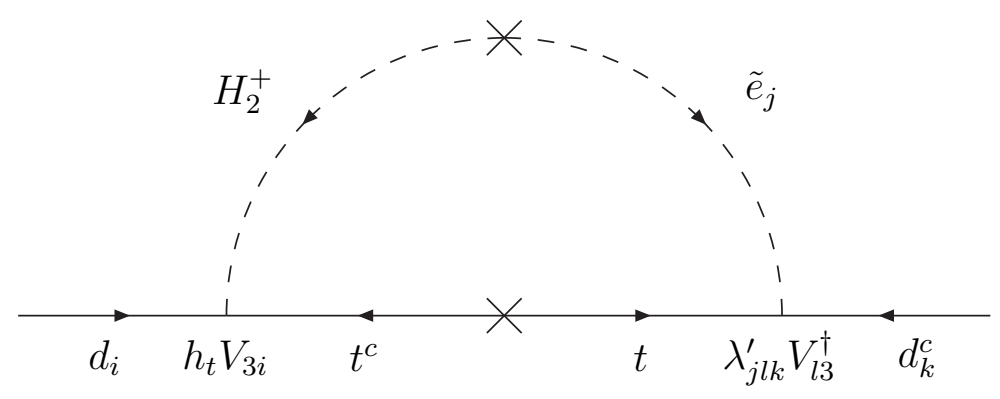

(c)

FIG. 2. Most important diagrams for the down-type quark EDM. There exist also diagrams similar to FIG. 1b, and also a supersymmetric counterpart of (c). 


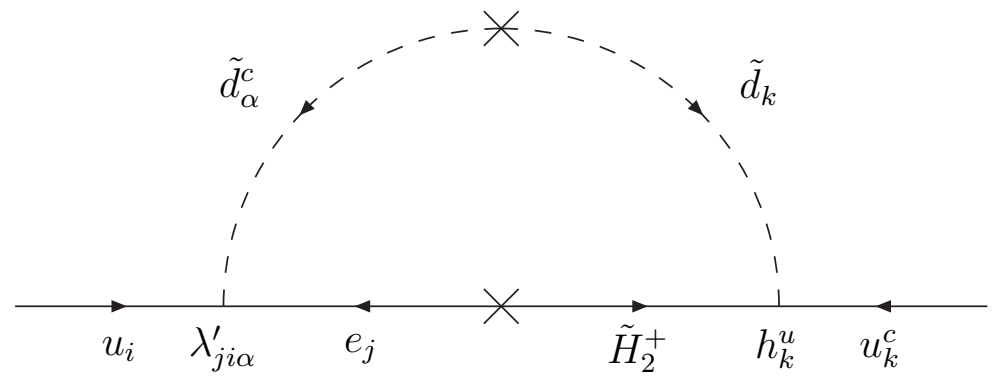

FIG. 3. Leading diagram for the up-type quark EDM. There is also a supersymmetric counterpart of this.

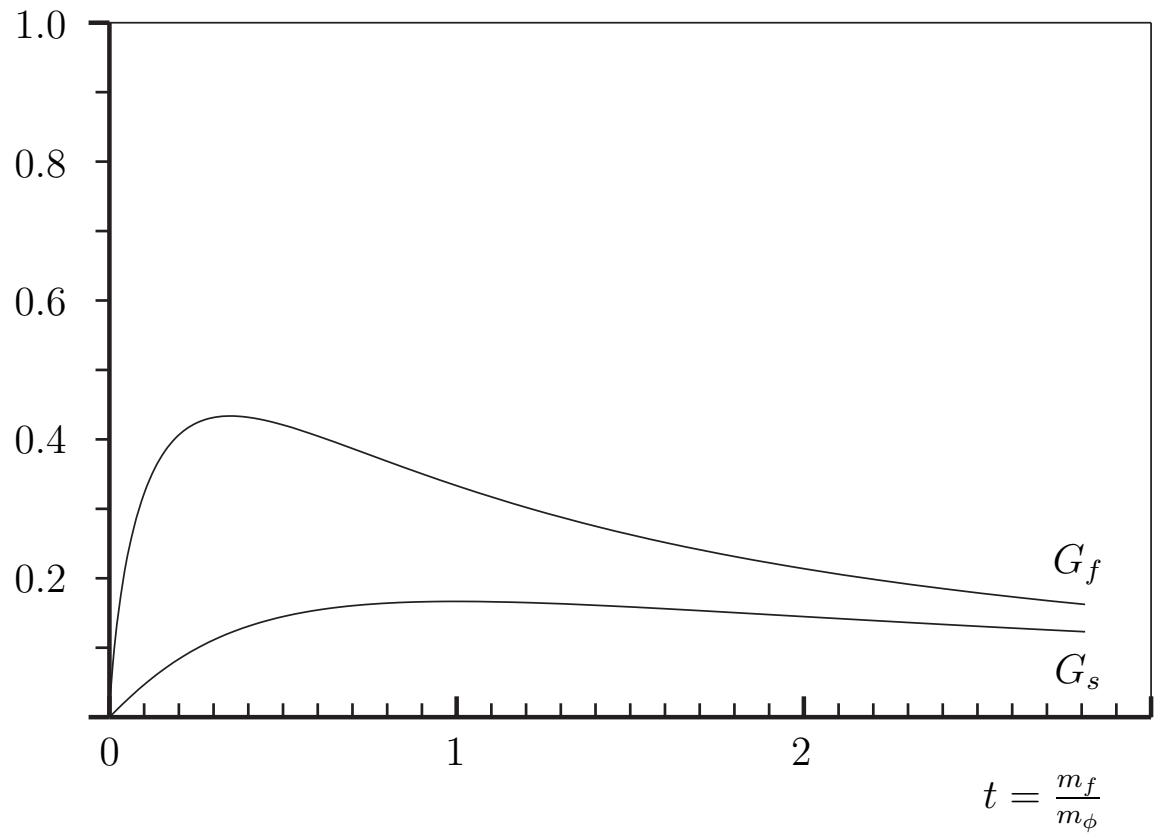

FIG. 4. Loop functions $G_{f}\left(m_{f} ; m_{\alpha}\right)$ and $G_{s}\left(m_{f} ; m_{\alpha}\right)$ as a function of $t=m_{f} / m_{\alpha}$ 